\title{
Remote Sensing of Precipitation using Reflected GNSS Signals: Response Analysis of Polarimetric Observations
}

\author{
Milad Asgarimehr, Mostafa Hoseini, Maximilian Semmling, Markus Ramatschi, Adriano Camps, \\ Hossein Nahavandchi, Rüdiger Haas, and Jens Wickert
}

\begin{abstract}
For the first time, rain effects on the polarimetric observations of the Global Navigation Satellite System Reflectometry (GNSS-R) are investigated. The physical feasibility of tracking the modifications in the surface roughness by rain splash and the surface salinity by the accumulation of freshwater is theoretically discussed. An empirical analysis is carried out using measurements of a coastal GNSS-R station with two side-looking antennas in right and left-handed circular polarization (RHCP and LHCP). Discernible drops in RHCP and LHCP powers are observed during rain over a calm sea. The power drop becomes larger in size at higher elevation angles. The average LHCP power drops by $\approx 5 \mathrm{~dB}$ at an elevation angle of $45^{\circ}$. The amplitude of the correlation sum shows a dampening, responding to rain rate systematically. The LHCP observations show higher sensitivity to rainfall compared to RHCP observations. The retrieved standard deviation of surface heights shows a steady increase over the rain rate. The derived surface salinity shows a decrease at rains higher than $10 \mathrm{~mm} / \mathrm{h}$. This study confirms the potential under environmental conditions of the GNSS-R groundbased station, e.g. with salinity mostly lower than $30 \mathrm{psu}$, over a calm sea, being a starting point for future investigations.
\end{abstract}

Index Terms-GNSS-Reflectometry, Rain, Polarimetric observations, surface-roughening, Sea surface salinity

\section{INTRODUCTION}

$\mathbf{T}$ He dependency of human beings on precipitation as a freshwater resource is clear. This component of the water cycle plays a key role in the economy and sustainable developments. Scarce or extreme rainfalls can lead to droughts or floods, threatening lives and properties. Besides, global warming is expected to change the extreme precipitation patterns in terms of magnitude and frequency [1]. Monitoring global precipitation

This research was funded by the the German Research Centre for Geoscineces GFZ in Potsdam, Germany. (Corresponding author: Milad Asgarimehr.)

Milad Asgarimehr and Markus Ramatschi are with the German Research Centre for Geosciences GFZ, 14473 Potsdam, Germany (e-mail: milad.asgarimehr@gfz-potsdam.de).

Mostafa Hoseini and Hossein Nahavandchi are with the Department of Civil and Environmental Engineering, Norwegian University of Science and Technology, 7491 Trondheim, Norway

Maximilian Semmling is with the Institute for Solar-Terrestrial Physics, German Aerospace Center (DLR-SO), D-17235 Neustrelitz, Germany.

Adriano Camps is with CommSensLab-UPC, Department of Signal Theory and Communications, UPC BarcelonaTech, c/Jordi Girona 1-3, 08034 Barcelona, Spain and Institut d'Estudis Espacials de Catalunya-IEEC/CTEUPC, Gran Capità, 2-4, Edifici Nexus, despatx 201, 08034 Barcelona, Spain

Rüdiger Haas is with the Department of Space, Earth and Environment, Chalmers University of Technology, 41296 Gothenburg, Sweden.

Jens Wikcert is with the German Research Centre for Geosciences GFZ 14473 Potsdam, Germany and Institute of Geodesy and Geoinformation Science, Technische Universität Berlin, 10623 Berlin, Germany. events can assist scientists to better recognize the climate change patterns.

In situ measurements and weather radars are the traditional methods to measure precipitation. They provide the required precipitation information for regional-scale studies, where a dense network of these instruments is established. These techniques are not able to capture the global precipitation due to the coverage limitations, especially over oceans and regions with underdeveloped infrastructures. To this end, meteorological satellites are used which are mainly equipped with advanced microwave and infrared instruments. The Tropical Rainfall Measuring Mission (TRMM) was one of the key satellites that contributed to improve our knowledge on the distribution and variability of precipitation within the tropics, operating from 1997 to 2015 [2]. The Global Precipitation Measurement (GPM) mission refers to a network of next-generation satellites providing precipitation information [3]. The GPM core observatory was launched in 2014 carrying a microwave radiometer The GPM centers bring precipitation observations from the operational satellites together and provide the standard data products. In [4], a review of the precipitation data sources and instruments is provided.

Exploitation of reflected Global Navigation Satellite System (GNSS) signals from the Earth's surface, so-called GNSSReflectometry (GNSS-R), has emerged as a powerful technique to obtain a variety of geophysical parameters and surface properties, see e.g. [5]. The GNSS-R technique is a multistatic radar method using existing signals from numerous GNSS satellites as the transmitters. The small and cost-effective receivers can be implemented at ground-based stations or onboard different air/spaceborne platforms such as satellites. The Cyclone GNSS (CYGNSS) is a constellation of eight Low Earth Orbiting (LEO) microsatellites, launched in December 2016, fully dedicated to GNSS-R [6]. Given the proven capabilities of GNSS-R to obtain a variety of surface and atmospheric parameters, precipitation monitoring can be a novel application of GNSS-R, which still needs investigations to enhance the knowledge on interactions between rain and the air-sea interface and consequently their impact on GNSS-R measurements. Due to the novelty of the technique, a very limited number of studies discuss the rain effects on GNSS-R observations, and this process is not yet well understood.

The precipitation signature was firstly seen in the measurements of the TechDemoSat-1 (TDS-1) satellite [7]. Based on a recent scattering model proposed in [8], the authors 
characterized the rain splash, the altered ocean roughness by the raindrops impinging on the surface, as a possible phenomenon reducing the received signal power at low wind speeds. This was also in agreement with the study discussing the roughness change as an increment of the sea surface slopes variance [9]. Later, a similar signature was also reported in CYGNSS measurements with the same explanation for the rain effects at low wind speeds [10]. At high winds an underestimation of wind speed using CYGNSS measurements is reported which could be potentially due to the damping effect of rain on larger scale surface waves [11]. In a simulation study, it is shown that the atmospheric attenuation by raindrops is insignificant in space-borne GNSS-R L-band measurements [12]. This type of effect is expected to be even smaller in magnitude in groundbased GNSS-R observations (such as those in this study) due to the much shorter distance between the specular point and the receiver.

The previous studies have investigated the rain impact on GNSS-R measurements obtained by spaceborne receivers with nadir-looking left-handed circular polarization (LHCP) antennas. The impact on the scattered GNSS signals in righthanded circular polarization (RHCP) is still unknown. The depolarization effect by the flattening of the heavy precipitation is shown in polarimetric GNSS Radio Occultation measurements [13], but this type of effect in polarimetric GNSS-R data is not yet studied.

The objective of this study is not only to investigate the rain effects on polarimetric observations, but also to further characterize the geophysical signatures and the interactions between rain and sea surface. Due to the lack of spaceborne polarimetric measurements, this study focuses on an groundbased experiment which can be potentially scaled up to spaceborne investigations in future. Section II discusses the physical theory and additionally, based on simulations, describes how the Sea Surface Salinity (SSS) change due to rainfall could be potentially detectable using polarimetric observations. Section III explains the used dataset for the empirical study. Section IV reports on the analysis, and finally Section V summarizes the results and gives the concluding remarks.

\section{PHYSICAL THEORY}

\section{A. Forward models}

The GNSS signals are originally transmitted in RHCP. Reaching the sea surface, part of the signals are bounced off in LHCP, while the rest keep their RHCP. The polarization ratio depends on the reflection geometry and sea surface permittivity. At elevation angles larger than the Brewster angle, the LHCP signal is dominant. If the reflection is recorded at an elevation angle lower than the Brewster angle, majority of the signal remains at RHCP. Forward models, being valid only for groundbased observations, describe reflected RHCP and LHCP powers, $P_{R H C P}^{r}$ and $P_{L H C P}^{r}$ respectively [14]:

$$
\begin{gathered}
P_{R H C P}^{r}=G_{R H C P}^{r e f}\left|\Re_{R R}\right| W^{2} L^{2} P_{0}, \\
P_{L H C P}^{r}=G_{L H C P}^{r e f}\left|\Re_{R L}\right| W^{2} L^{2} P_{0}
\end{gathered}
$$

where $G_{R H C P}$ and $G_{L H C P}$ are the RHCP and LHCP antenna gains, $P_{0}$ is the incoming reference power at the receiver position, and $W$ and $L$ are the power loss due to insufficient delay-Doppler tracking of the reflected signal and surface roughness. Finally, $\mathfrak{R}$ is the polarization-dependent Fresnel reflection coefficient. Analogous to the polarization of incoming direct signals, there are two Fresnel coefficients. Co-polar coefficients, $\mathfrak{R}_{R R}$ and $\mathfrak{R}_{L L}$, and cross-polar coefficients, $\mathfrak{R}_{R L}$ and $\mathfrak{R}_{L R}$, can be obtained from the complex dielectric permittivity of sea surface water $\epsilon$, and local elevation angle $\theta$ as follows [15]:

$$
\begin{aligned}
& \mathfrak{R}_{R R}=\mathfrak{R}_{L L}=\frac{1}{2}\left(\mathfrak{R}_{V V}+\mathfrak{R}_{H H}\right), \\
& \mathfrak{R}_{R L}=\mathfrak{R}_{L R}=\frac{1}{2}\left(\mathfrak{R}_{V V}-\mathfrak{R}_{H H}\right) .
\end{aligned}
$$

In the above equations, $\mathfrak{R}_{V V}$ and $\mathfrak{R}_{H H}$, polarization components parallel and perpendicular to the incidence plane, read

$$
\begin{gathered}
\mathfrak{R}_{V V}=\frac{\epsilon \sin \theta-\sqrt{\epsilon-\cos ^{2} \theta}}{\epsilon \sin \theta+\sqrt{\epsilon-\cos ^{2} \theta}}, \\
\mathfrak{R}_{H H}=\frac{\sin \theta-\sqrt{\epsilon-\cos ^{2} \theta}}{\sin \theta+\sqrt{\epsilon-\cos ^{2} \theta}} .
\end{gathered}
$$

Since $P_{0}$ is here unknown, we introduce the power ratios $P_{R H C P}$ and $P_{L H C P}$ as the observables:

$$
\begin{aligned}
P_{R H C P} & =P_{R H C P}^{r} / P^{d} \\
& =\left(G_{R H C P} / G^{d}\right)\left|\Re_{R R}\right|^{2} L^{2}, \\
P_{L H C P} & =P_{L H C P}^{r} / P^{d} \\
& =\left(G_{L H C P} / G^{d}\right)\left|\Re_{R L}\right|^{2} L^{2}
\end{aligned}
$$

where $P_{0}$ is cancelled out being divided by the power of direct signal $P^{d}=G_{d} P_{0}$.

\section{B. Salinity change}

As Equations 5 and 6 imply, Fresnel coefficients are dependent on the relative permittivity of the seawater and the elevation angle of the signals. The permittivity of seawater is in turn controlled by the SSS and Sea Surface Temperature (SST). The permittivity value can be obtained using models relying on the L-band measurements [16], [17], [18]. These models are used in ocean state retrievals using GNSS-R measurements [19]. Figure 1 shows how SSS and SST changes can alter permittivity value based on simulations using the model proposed in [18]. As shown, at SSS values lower than almost $30 \mathrm{psu}$, which is the SSS range in the area studied in the following sections, the SSS has a more significant role in controlling the permittivity value. The calculations using the Klein-Swift dielectric constant model confirm that the sensitivity of emissivity, and therefore reflectivity, to SST at L-band is insignificant also at higher SSS values and SSTs lower than $15^{\circ} \mathrm{C}$ [20].

Conditions of sea surface salinity (SSS), considered in the following simulations, apply for the Western Baltic Sea where long-term GNSS-R measurements were conducted at the Onsala Observatory, discussed in the following sections. The historical observations of a station about 29 kilometers away, between 2001 and 2009, show that there are large SSS variations around 25 psu remaining below 30 most of a year. 

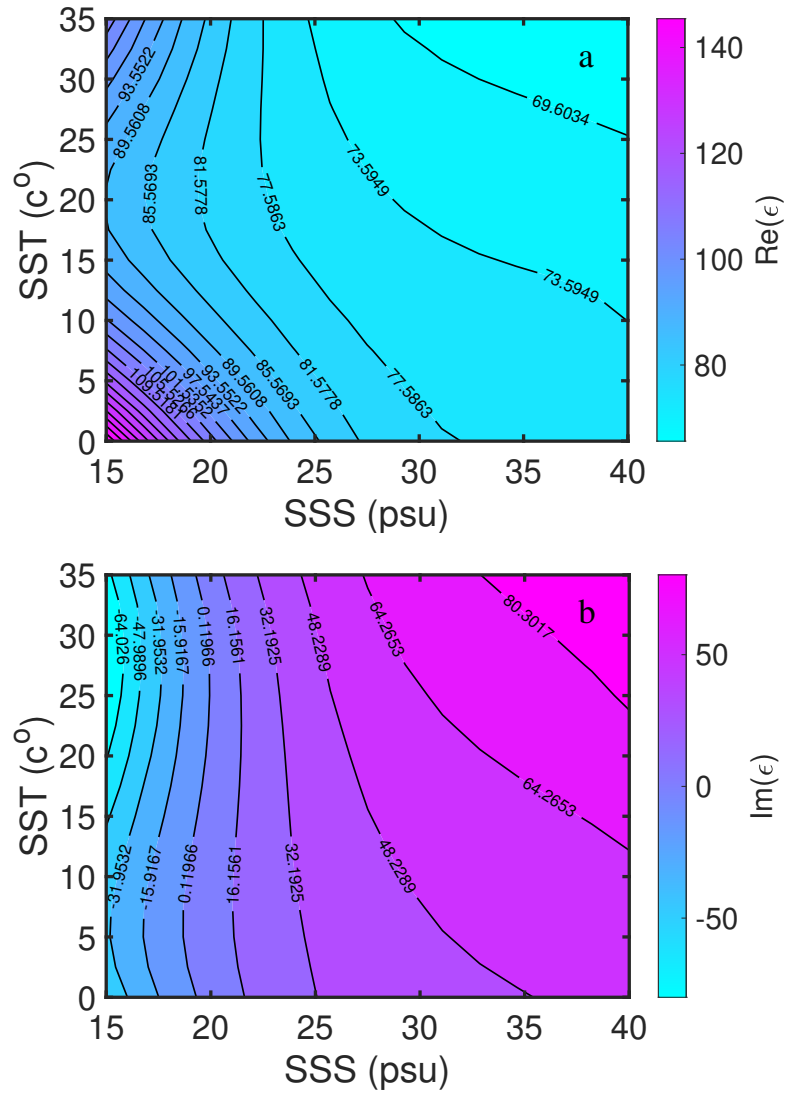

Fig. 1: Real (a) and imaginary (b) parts of dielectric permittivity of sea water vs. Sea Surface Salinity (SSS) and Sea Surface Temperature (SST).

Rain creates a fresher layer of water accumulating on the sea surface. This causes a rapid change in the SSS. The depth of the freshwater layer evolves, increasing or decreasing as a function of rain accumulation rate and sea state mixing the water. The mixing rate is in turn dependent on surface wind speed. So, more significant SSS changes are expected over calm ocean, i.e. at low wind speeds. This has been also the condition providing the means to detect roughness change by rainfall, as discussed in [7].

Using a forward Rain Impact Model (RIM), the SSS change is predictable as follows [21]:

$$
\begin{aligned}
S_{R I M}= & S_{0}\left[\left(\prod_{i=1}^{n}\left[1+\frac{R_{1 i}}{\sqrt{k_{z} * t_{i}}} e^{-z^{2} /\left(4 k_{z} t_{i}\right)}\right]\right)\right]^{-1} \\
& *\left[1+\frac{R_{2}}{\sqrt{k_{z} * t}} e^{-z^{2} /\left(4 k_{z} t\right)}\right]^{-1}
\end{aligned}
$$

where $S_{R I M}$ is the new salinity after precipitation, $z$ is the depth in meters, $n$ is the total number of rain events, $S_{0}$ is the initial salinity in psu, $t$ is time in seconds, $k_{z}$ is the vertical eddy diffusivity coefficient, $R_{1 i}$ and $R_{2}$ are the rain surface impulse function, the rain accumulation integrated over the characteristic mixing depth, for each event in meters. It is shown that the skin depth at the L-band frequency $(1.4 \mathrm{GHz})$ and a fixed water temperature $\left(20^{\circ} \mathrm{C}\right)$ and salinity $(34 \mathrm{psu})$ is

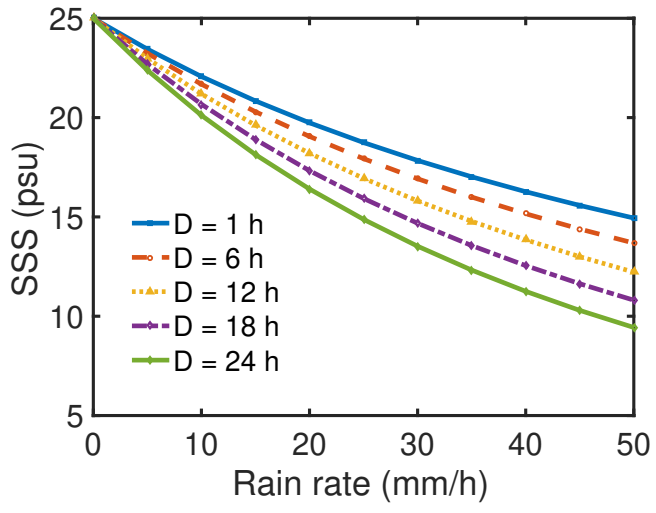

Fig. 2: Sea Surface Salinity (SSS) vs. rain rate at different duration lengths (D) and $S_{0}=25$ psu.

$0.955 \mathrm{~cm}$ [22]. By definition, the skin depth is the medium thickness through which the electric field amplitude of the propagating electromagnetic wave falls to $1 / e$, i.e. $37 \%$, of its original value. Accordingly, the L-band penetration depth is not more than $1 \mathrm{~cm}$, and we consider $z=0.005 \mathrm{~m}$ in this study.

Fig. 2 shows the SSS change due to rain event at different rates and duration $(1,6,12,18$, and 24 hours). For instance, the blue curve shows an 1-hour rainfall at a constant rate of $10 \mathrm{~mm} / \mathrm{h}$ drops the SSS from $25 \mathrm{psu}$ to $\approx 24 \mathrm{psu}$. Following that, Fig. 3 shows the expected power levels and their ratio in these rain event scenarios. As demonstrated, altered SSS by rain could affect the observations at significant rain rates with a long enough duration. Besides, the power change for both RHCP and LHCP reflected GNSS signals and their ratio at different SSS values are illustrated in Fig. 4. As shown, significant drops of SSS are distinguishable in the power changes. This phenomenon increases the power of the LHCP signals, whereas, it decreases that scattered in RHCP. The discrepancy in the LHCP to RHCP power ratio will be therefore more significant leading to a more detectable SSS signature. This can be an advantage of polarimetric GNSS-R observations.

The RIM used here considers the surface salinity profile is a function of depth and time. Wind speed is not included and the model is assumed to describe the near-surface salinity profile at low wind speeds $(0-3 \mathrm{~m} / \mathrm{s})$. The analysis in the following sections is conducted at calm sea state. This is the condition in which the model is valid. Nevertheless, we should consider a level of uncertainty at different wind speeds. Besides, the wind might have a weaker effect in the coastal areas due to the land sheltering and the limited fetch [23], leading to a faster accumulation of the freshwater.

\section{Roughness change}

When a raindrop impinges on the sea surface, a cavity with a crown is firstly created which will change to a vertical stalk at the center of rings of gravity-capillary waves. These waves propagate outwards, known as "ring waves" [24]. These smallscale waves alter the surface roughness which could affect the scatterometric measurements. 

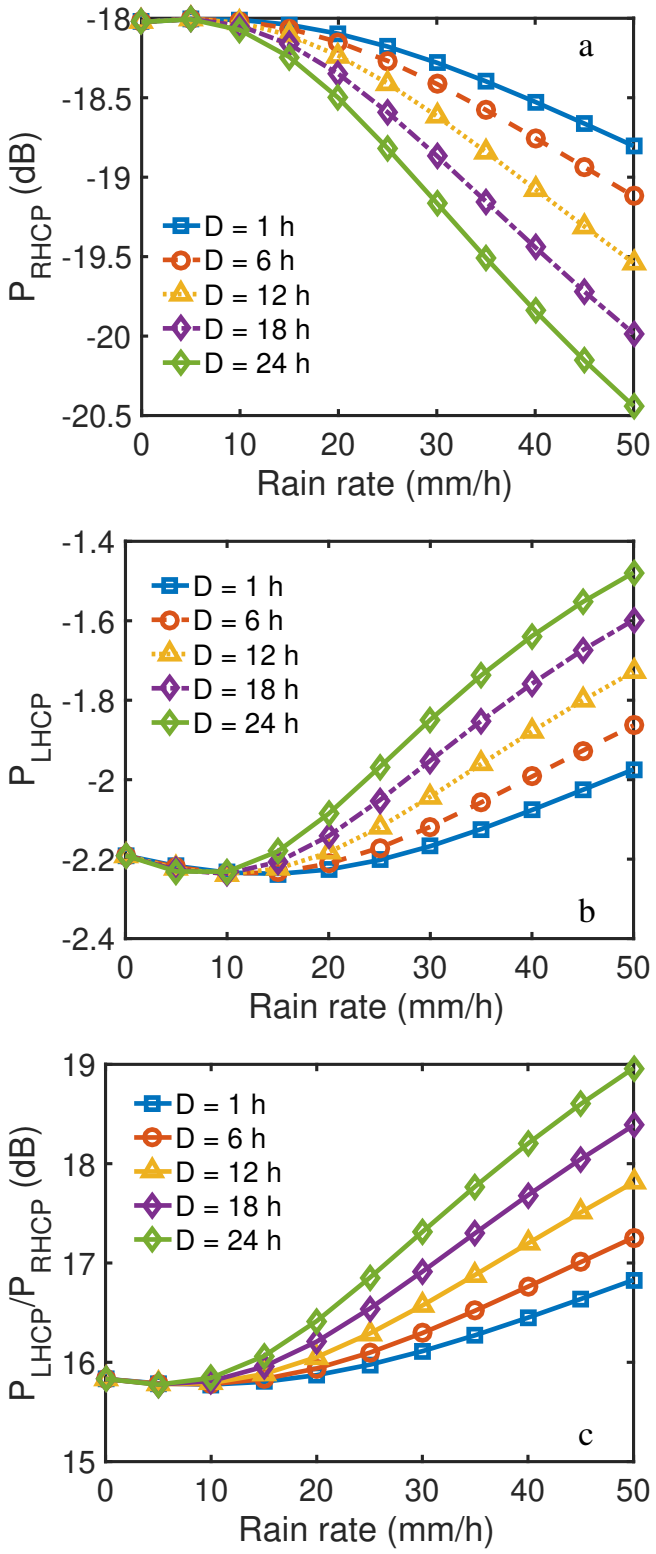

Fig. 3: The power of reflected RCHP (a) and RHCP (b) signals and their ratio (c), and $S S T=10^{\circ} \mathrm{C}$ vs. rain rate at different rainfall duration lengths $\mathrm{D}, \theta=30^{\circ}$, and $S_{0}=25$ psu.

There are numerous laboratory experiments investigating the altered surface waves by rain. Based on them, theoretical models have been proposed to describe the generated waves and the impact on the surface, see e.g. [25], [26], [27], [28]. Although the ring waves intensify the centimeter-scale roughness, rain could also attenuate ocean surface gravity waves [27].

The developed theories based on laboratory experiments do not yet sufficiently answer the questions on the mechanism of rain impact on ocean surface waves in real environments [29]. The simulations based on GNSS signal scattering models and the log Gaussian spectrum of rain-generated waves [28], do not show an exact match with the rain impacts observed in the empirical measurements [7], however, the authors did not
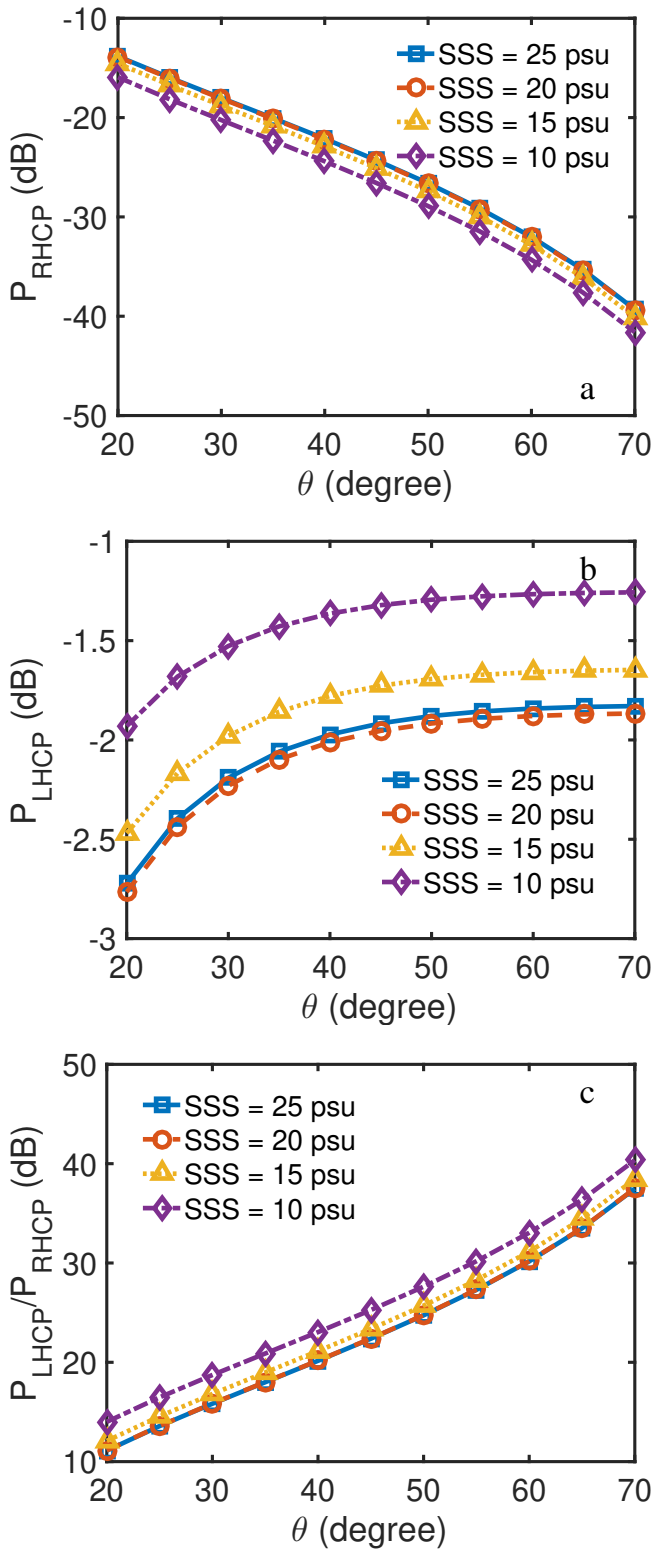

Fig. 4: The power of reflected RCHP (a) and RHCP (b) signals and their ratio (c) vs elevation angle $\theta$ at different Sea Surface Salinity (SSS) and $S S T=10^{\circ} \mathrm{C}$.

exclude the possibility of other effects existence such as swell and downdraft. In a recent study, field observations are used to describe rain impact on surface roughness [29]. Therein, more significant rain-enhanced gravity-capillary waves, with wavelengths smaller than $\lambda=56 \mathrm{~mm}$ are reported. These waves control the intensity of forward GNSS scattering in the regime of weak diffuse scattering, i.e. at low wind speed, which is the environmental condition for observing altered roughness effects in GNSS-R observations.

Further studies are required to characterize the rain-ocean interactions and consequently the effects on signal forward scattering patterns. We should therefore admit the effect of rain on the ocean surface is one of the least understood processes. We will discuss the result of this study on the surface change 
more in an empirical sense trying to enhance the knowledge on this process in a top-down approach.

In this study, the polarization-independent power loss due to the surface roughness is considered as [14]

$$
L=\exp \left[(-1 / 2)\left(4 \pi^{2} / \lambda^{2}\right) \sigma^{2} \sin ^{2} \theta\right]
$$

where $\sigma$ is the standard deviation of sea surface height and $\lambda$ is the wavelength of GNSS signal. It should be noted that the power loss model described here applies to coherent scattering and does not account for possible polarization-dependent signatures of the roughness. This means the reflected signals can exhibit some degrees of ellipticity originated from the structure of the sea surface roughness. For instance, the horizontal parallel crests can act as an oriented structure affecting the horizontal component of the signals. Such roughness-induced polarimetric effects need to be further investigated in future studies.

\section{EXPERIMENTAL DATA}

A GFZ coastal GNSS-R station at Onsala Space Observatory $\left(57.393^{\circ} \mathrm{N} ; 11.914^{\circ} \mathrm{E}\right)$ in Sweden is considered in this study for investigating the effect of precipitation on GNSS-R polarimetric observations. The dataset covers a period of one year from January to December 2016. The station is equipped with a GNSS Occultation, Reflectometry, and Scatterometry (GORS) receiver [30] with two reflectometry antennas in different polarization, i.e. RHCP and LHCP. The antennas are tilted $\approx 98^{\circ}$ with respect to the zenith. The boresight of the reflectometry antennas is set at azimuth angle of $\approx 150^{\circ}$ to capture sea surface reflections with the highest gain values. The station environment and antennas are shown in Fig. 5.

The gain pattern of the antennas for incoming signals as a function of satellite elevation and azimuth angles is shown in Fig. 6. The experiment setup assigns high gains to the signals at grazing elevation angles, i.e. signals from satellites at $0^{\circ}$ to about $40^{\circ}$ elevation angles. The signals from the satellites with azimuth angles close to the antennas boresight, i.e. $150^{\circ}$, are recorded at higher gains. The gain pattern is assumed to be equal for both of the RHCP and LHCP sea-looking antennas.

The receiver uses designated channels to track the signals of the up-looking antenna (master channel) and side-looking antennas (slave channels), as previously described by [31]. Inphase and Quadrature samples (I/Q) of the respective channels are recorded. Fig. 8 shows an example of the receiver output from the GPS satellite PRN 7 signals captured by the sealooking antennas.

The antennas, here, have a small baseline $(\approx 20 \mathrm{~cm})$ with respect to each other and are mounted with a height of about 3 $\mathrm{m}$ above the reflecting sea surface. For the given geometry the differential delay between direct and reflected signals cannot be resolved in the code delay domain. Multipath patterns of the direct and the reflected signals occur in the receiver output as previously explained, for example, by [32]. As shown in Fig. 8, a low-frequency pattern is evident in the interferometric pattern, which is attributed to the direct signal. A separation algorithm is applied to find the low-frequency pattern of the direct signal and the higher-frequency pattern of the reflected

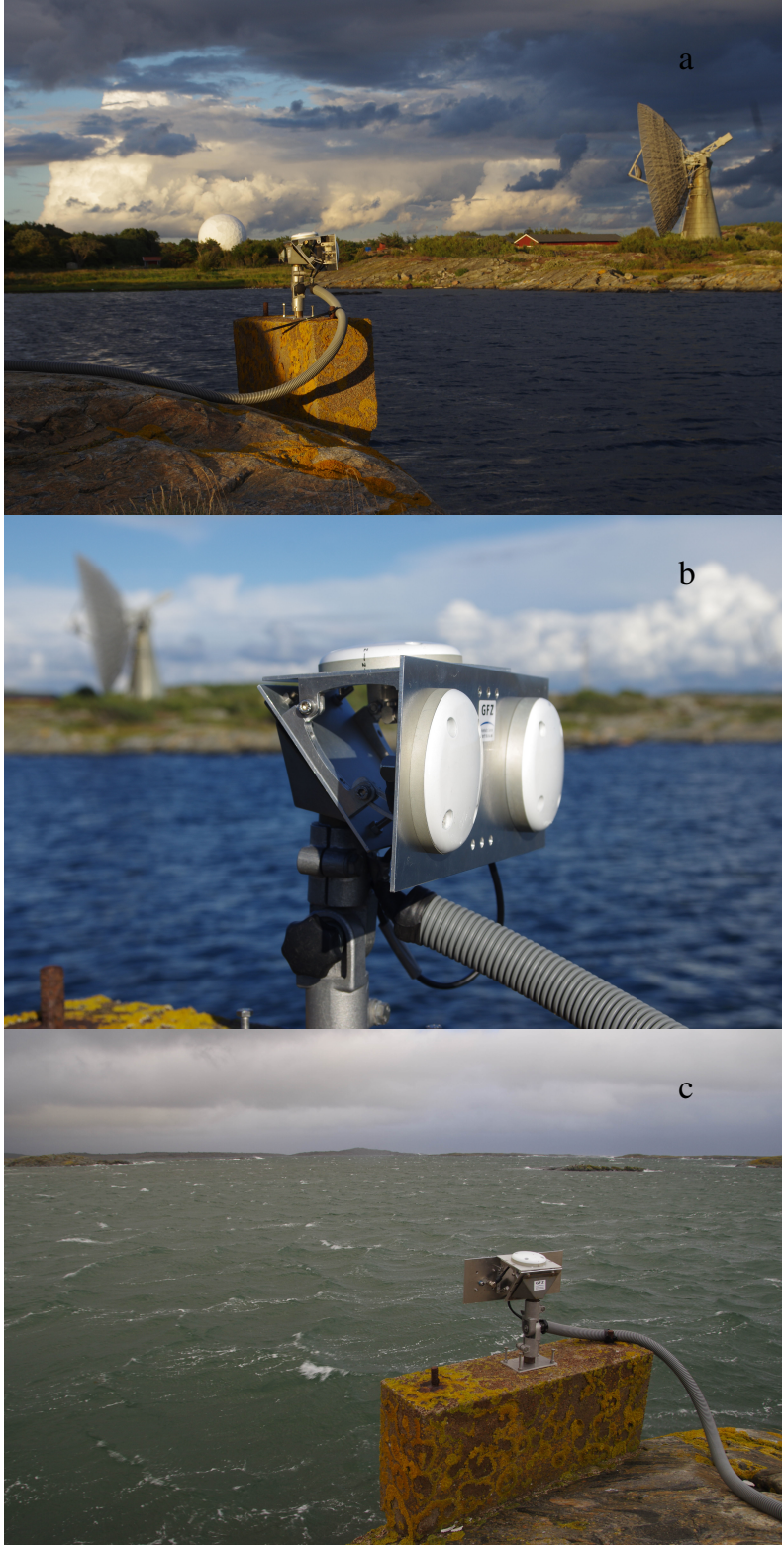

Fig. 5: An eastward view of the GFZ GNSS-R station at Onsala Space Observatory (a), the zenith looking, RHCP, and side looking, RHCP and LHCP, antennas (b), and the sea targeted at the antenna boresight (c).

signal, as described by [33]. To be more specific, a first-order polynomial is fitted to the low-frequency variations of I/Q correlation sum in each segment. Having the contribution of the direct signal from this fitting, the I/Q contributions of the reflected signal is determined. For the here given geometry the separation algorithm yields estimates of the direct and reflected signal power every ten minutes.

The experiment uses wind, tide gauge, and precipitation measurements as the ancillary information for the analysis The wind and sea-level measurements are obtained from the nearest meteorological and tide gauge stations, respectively. For the precipitation estimates, we use the GPM, half-hourly $0.1^{\circ} \times 0.1^{\circ}$ Version 06B Level 3 IMERG final run product.

The GNSS-R station can measure reflected signals at a 


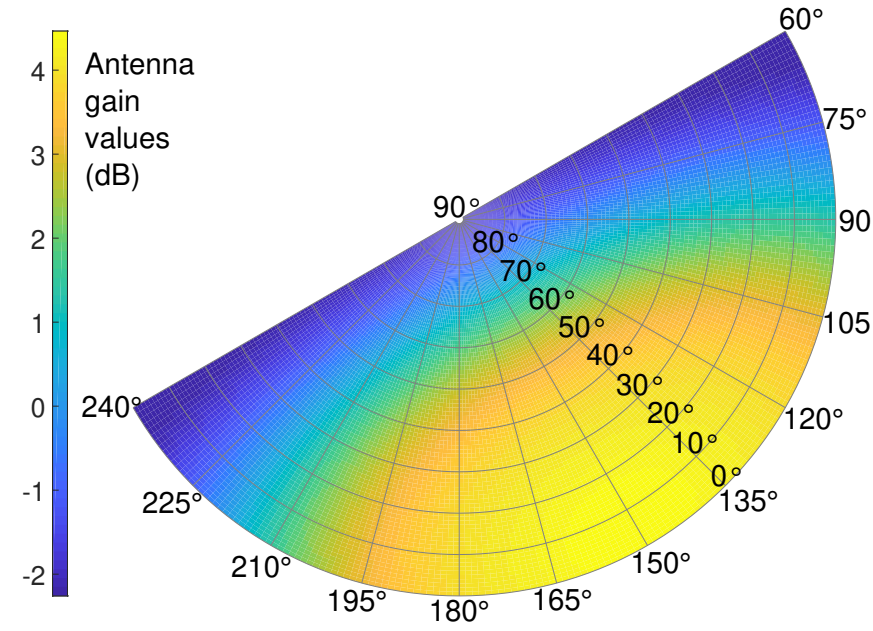

Fig. 6: Gain pattern of the reflectometry antennas as a function of satellite elevation and azimuth angles.

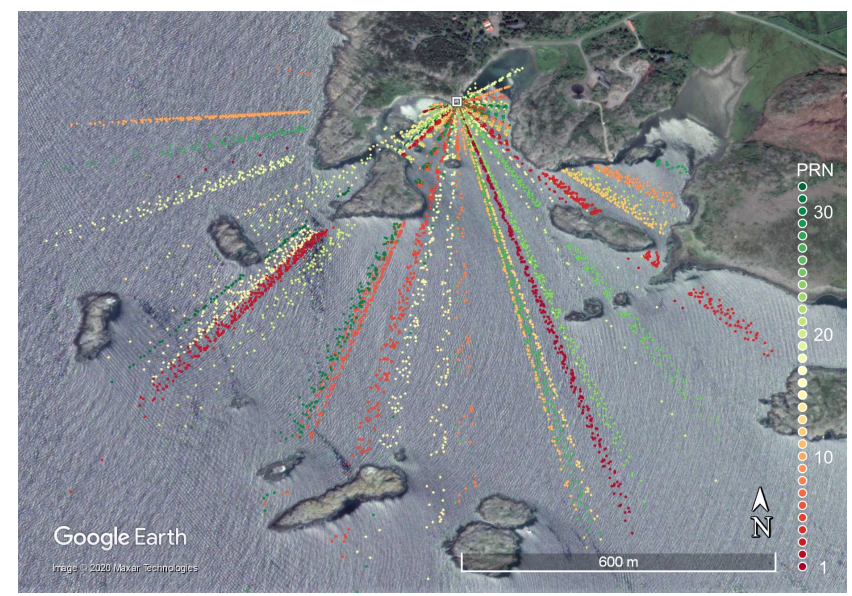

Fig. 7: The spatial extent of the specular points over the sea.

sampling rate of $0.1 \mathrm{~Hz}$ from GPS satellites with elevation angles ranging from $0^{\circ}$ to $50^{\circ}$. The spatial coverage of the specular points is shown in Fig. 7. During the one-year observation period, from January to December 2016, 175 178 measurements are recorded, from which 26413 data are captured during rainfall, i.e. at rain rates higher than $0 \mathrm{~mm} / \mathrm{h}$ The maximum and average recorded rain rates are 23.04 and $0.09 \mathrm{~mm} / \mathrm{h}$, respectively.

\section{ANALYSis}

Figure 9 visualizes the power in both LHCP and RHCP versus the elevation angle along with simulated measurements using Equations 1 and 2, derived from the entire dataset. There is a general agreement between the observed and simulated measurements, however, the discrepancy of $P_{R H C P}$ is larger compared to $P_{L H C P}$. Especially, there is a mismatch between the RHCP simulated and observed measurements at low elevation angles $\left(\lesssim 10^{\circ}\right)$.

To investigate rain effects, the data are limited to wind speeds lower than $5 \mathrm{~m} / \mathrm{s}$ as it is the necessary condition for detecting rain splash. Additionally, only the data at winds blowing from the land side, i.e. with azimuths between $0^{\circ}$ and $150^{\circ}$, are considered. The location of the station and the considered azimuth range is shown in Figure 10. In this condition, the wind-wave generation is limited by the short fetch. We use this sheltering effect of the coastline on the nearshore waves to further exclude wind associated effects in the measurements. Then, this coastal experiment better provides the environmental conditions for tracking the rain splash effects compared to those using spaceborne measurements. In [23], the authors report on the insignificant correlation between the offshore or land breeze and sea surface roughness at this GNSS-R station owing to the limited fetch. Furthermore, no meaningful correlation between precipitation and wind speed could be identified in our statistical analysis. The correlation coefficient between precipitation and wind speed is 0.1 .

Figure 11 demonstrates the power of the reflected signal in both polarizations, in different cases, during rainfalls and rain-free measurements. A significant discrepancy between the two cases is shown which is larger at higher elevation angles. Besides, the simulations show the power behavior at different surface states. The similar patterns between the simulated and observed behavior show a possibility that the observed effects appear due to the rain splash altering the surface state, as discussed in [7]. In that spaceborne analysis, studying the intensity of the effect at different incidence angles was postponed to a future work due to the uncertainty of measurements. Here, the dependency of the rain effect on the reflection geometry is evident. We cannot exclude that the power drop in the empirical data might be intensified by other type of effects such as swell and downdraft which can be potentially the factors causing larger standard deviations during rainfall in Figure 11. According to the figure, a higher level of sensitivity to rain in LHCP measurements is seen compared to those RHCP observations. Besides, the Probability Density Function (PDF) of the power measurements, derived as the kernel density estimation (kde) is given in Figure 12. It indicates that rain has distorted the PDF in both sets of measurements, dampening the peak and increasing the probability at lower values of power.

Figure 13 shows the RHCP and LHCP reflection amplitudes at two different precipitation conditions during the setting period of the GPS satellite PRN 7. The shown exemplary cases do not necessarily meet the aforementioned conditions on the wind speed and its azimuth. Hence, wind speed and direction profiles are additionally given. The left column in the figure shows the I/Q components of the reflected signal during a rainfree period. As expected, a general trend of decreasing RHCP amplitude, and on the contrary, increasing LHCP amplitude over the satellite elevation angle is evident, which is due to the reflection geometry. Both cases are associated with offshore winds, so we expect to have a minimized wind-driven roughness effect. Figure 13(a) on the left column reports on slightly higher wind speed. As a result, an insignificantly rougher sea surface with more power loss could be anticipated for the left column. The right column shows the case with the same GPS satellite and reflection geometry but during a rain-affected period.

Although the wind conditions might imply larger amplitude in rain-affected case, the RHCP and LHCP correlation sums 

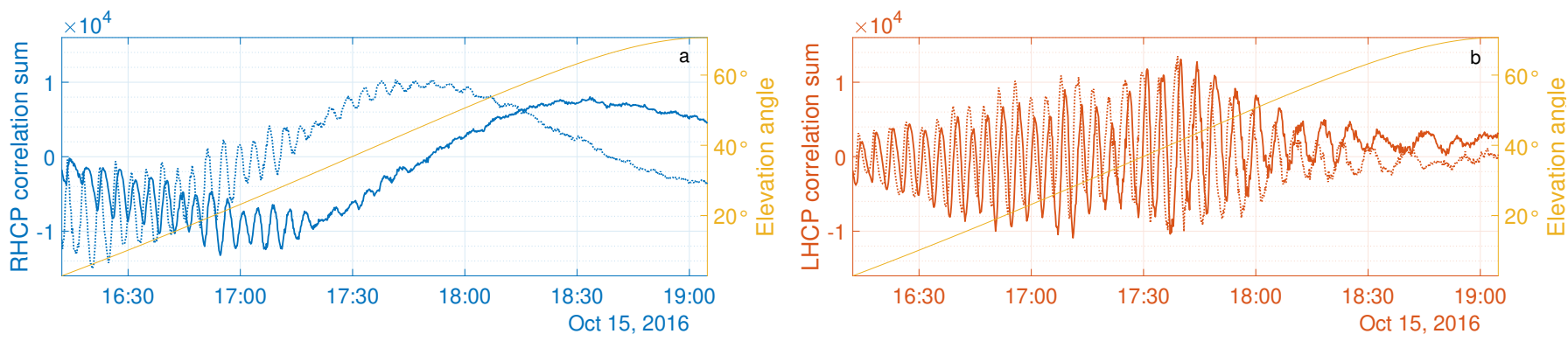

Fig. 8: Reflectometry observations of the GPS satellite PRN 7 on October 15, 2016 obtained from the Onsala station using two sea-looking antennas with Right- and Left-handed circular Polarizations (RHCP and LHCP). The receiver output, i.e. the correlation sums, at in-phase and quadrature channels are shown as solid and dotted lines, respectively.
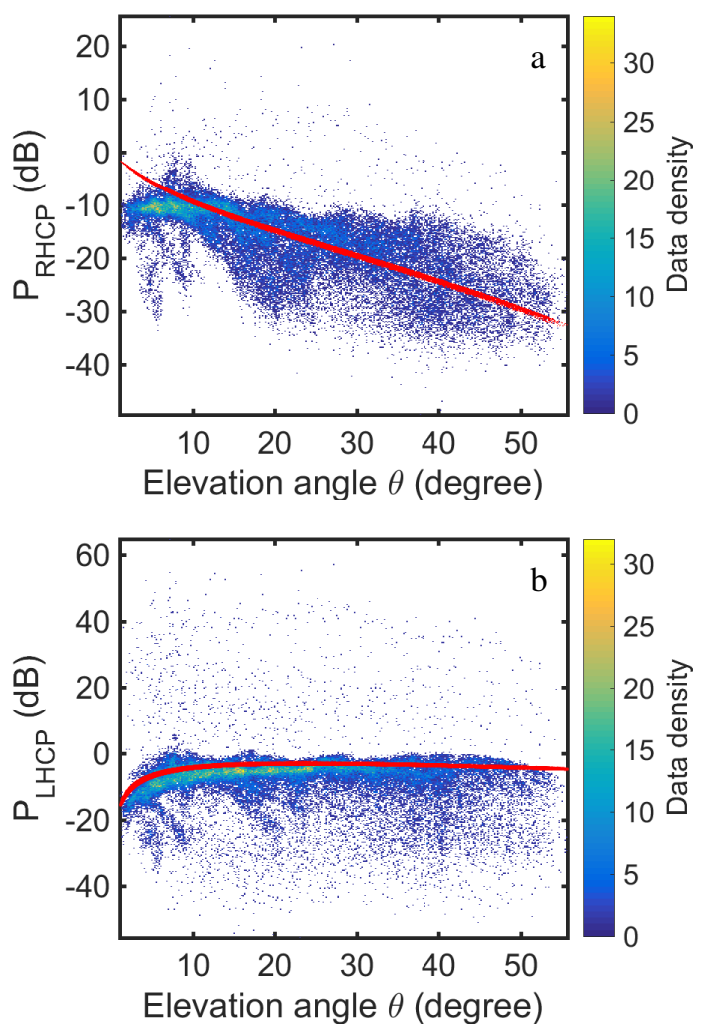

Fig. 9: The power of reflected RCHP (a) and LHCP (b) signals vs elevation angle $\theta$ along with simulated observations at $\sigma=$ $0.03 \mathrm{~m}$ in red (entire dataset). The SST values are obtained from a nearby meteorological station. The SSS for each observation is estimated as an average value based on historical records of between 2001 and 2009 at a station located about 29 kilometers away.

in Figure 13(d) and (f), exhibit smaller amplitudes leading to lower power ratios in Figure 13(h). This power loss in the measurements of both polarizations compared to those in $13(\mathrm{~g})$ can be explained with the rainfall. The striking fact is the systematic response to the rain rate in Figure 13(h). There is an increase in the rain rate, from almost $0.5 \mathrm{~mm} / \mathrm{h}$ to $3.6 \mathrm{~mm} / \mathrm{h}$, at elevation angles between $17^{\circ}$ and $26^{\circ}$. The amplitudes shrink within this range along with a decrease in power ratios. The

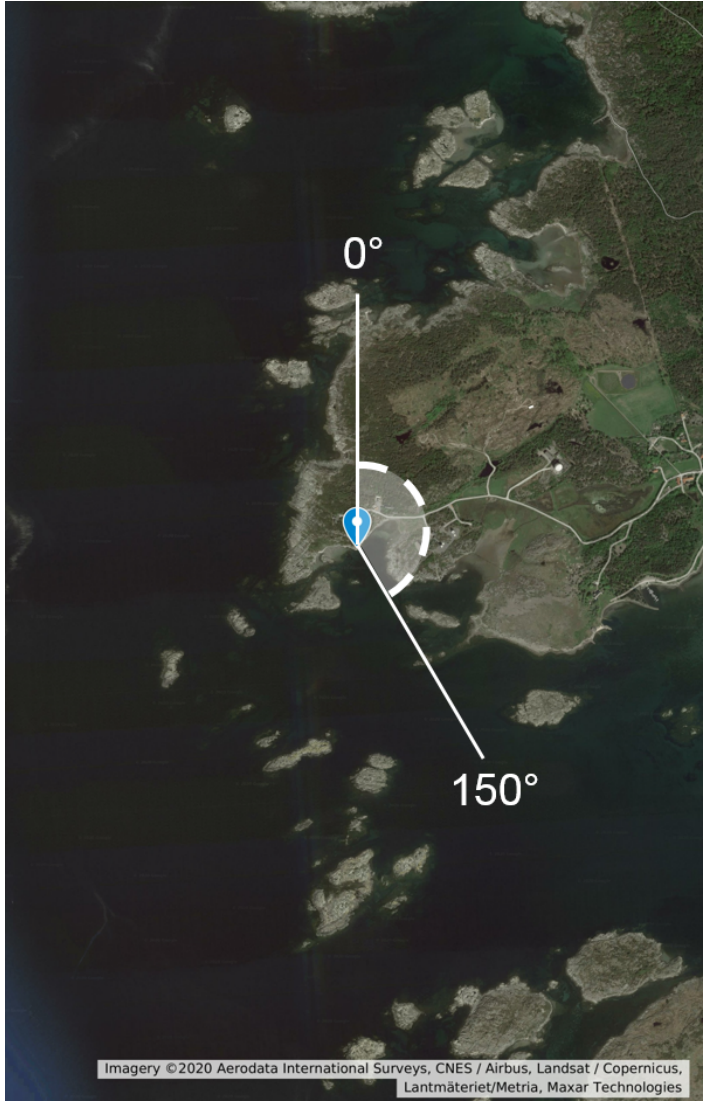

Fig. 10: The GNSS-R station location and the wind azimuth range condition $\left(0^{\circ}-150^{\circ}\right)$ in the analysis.

rain rate decrease also stimulates a relative increase in both of the power ratios when the satellite elevation angle passes $26^{\circ}$. However, the power ratios at elevation angles from $26^{\circ}$ to $50^{\circ}$ with a rain rate of $1.1 \mathrm{~mm} / \mathrm{h}$ are slightly lower compared to the power ratios in Figure 13(g). The amplitude change due to rain is more prominent in the LHCP observations compared to those in RHCP.

Considering the SSS change as the additional rain effect, we develop an inversion algorithm to estimate the standard deviation of surface heights and the SSS. $P_{R H C P}(\sigma, S)$ and $P_{L H C P}(\sigma, S)$ are considered as the observable parameters, see Eq. 7 and Eq. 8. The values of $\sigma$ and $S$ are sought which 

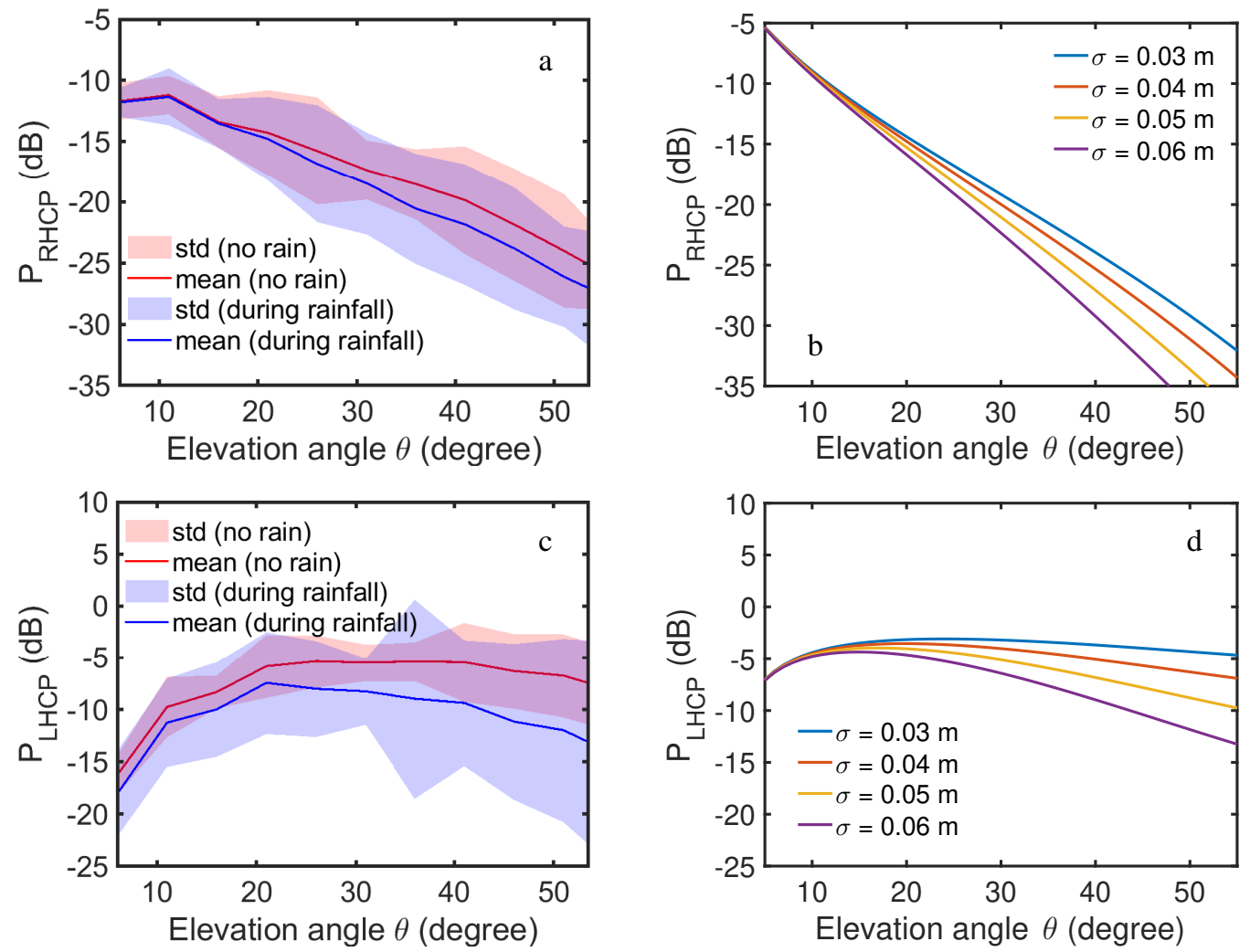

Fig. 11: The RHCP (a) and LHCP (c) power ratios and in different cases, during rain events, at rates higher than $0.2 \mathrm{~mm} / \mathrm{h}$, and at no rain along with model-simulated RHCP (b) and LHCP (d) power ratios at different standard deviations of surface heights $\sigma$. Average and maximum rain rates of the data during rainfall are 1.5 and $23.0 \mathrm{~mm} / \mathrm{h}$ respectively.

minimizes the cost function:

$$
\begin{aligned}
\delta(\sigma, S)= & \frac{1}{n} \sum_{i=1}^{n}\left|P_{R H C P, i}^{o}-P_{R H C P, i}^{s}(\sigma, S)\right| \\
& +\frac{1}{n} \sum_{i=1}^{n}\left|P_{L H C P, i}^{o}-P_{L H C P, i}^{s}(\sigma, S)\right|
\end{aligned}
$$

where the superscripts $o$ and $s$ indicate the observed or simulated parameters, respectively. We assume that the variations of $S$ and $\sigma$ within three hours are insignificant. So, these parameters are retrieved as the average value for every threehour time span and $n$ is the number of observations residing in each temporal bin. The conditions on the wind speed and its direction are applied here. Figure 14 shows the obtained values for both parameters as a function of the rain rate.

According to Fig. 14, most of the data has a $\sigma$ value of $\approx$ $3.5 \mathrm{~cm}$. A steady increase in the average $\sigma$ over the rain rate is observed with the correlation coefficient of 0.96 . The derived SSS values show no significant trend at rain rates lower than $10 \mathrm{~mm} / \mathrm{h}$. Most of the data points, in a rain-free condition, reside at the SSS of $\approx 26 \mathrm{psu}$. A slight downward trend in the SSS at low rain rates, between 0 and $2.5 \mathrm{~mm} / \mathrm{h}$, is observed, decreasing the average SSS to $\approx 24$ psu. Nevertheless, at higher rain rates up to $10 \mathrm{~mm} / \mathrm{h}$ no significant change is observed in the average SSS value, which could statistically approve the
SSS change due to rain. However, at rain rates larger than 10 $\mathrm{mm} / \mathrm{h}$ a significant drop in the SSS values is observed.

The altered surface state and the effects on the power ratios shown here are much larger than the theoretical study on the surface effect of rain based on laboratory measurements, e.g. [28]. There is also a quantitative mismatch on the observed effects in TDS- 1 and CYGNSS measurements with the theoretical studies [7], [10]. This inconsistency could be, at least partially, associated with the environmental differences between laboratory and real sea conditions. In a laboratory, the theoretical knowledge is derived based on observing wave parameters in a water tank influenced by artificially-generated rain and wind. In [29], the authors discuss that the variation in such condition is spatial, and not temporal. This is a considerable difference in a real sea, where rain may affect the surface state rapidly, but environmental conditions do not vary spatially at a significant level for long periods of time. As a result, the laboratory-derived models are not satisfactory to characterize the problem of rain splash impacts on the sea surface waves and the change in the GNSS forward scattering patterns. In situ measurements of the impact of rain show a strong increase in the roughness in the scale of the gravity capillary waves with a wavelength between 5.4 and $56 \mathrm{~mm}$ [29]. These waves control the intensity of the reflection when the coherent component is considerable, i.e. when the wind speed is low and the Rayleigh parameter is large enough, see [7]. This is the condition applied to the analysis here. 

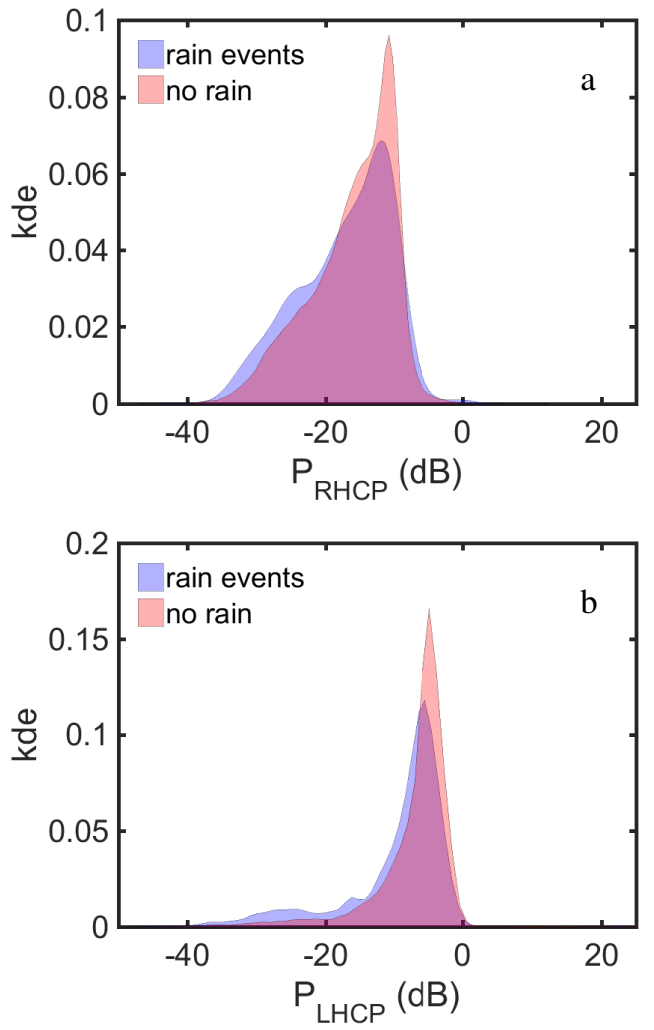

Fig. 12: Probability density function of RCHP (a) and LHCP (b) power in cases during rainfall and at no rain, derived as kernel density estimation (kde).

The discussions in Section II theoretically proves the potential of simultaneous retrieval of SSS and surface state using polarimetric observations. The average values in Fig. 14 demonstrate the rain-associated systematic behaviors of these parameters. However, considerable uncertainties in $\sigma$ and the SSS change are also seen which could be associated with different factors.

We cannot exclude non-rain environmental changes, such as swell and downdraft, could still affect the retrievals and increase the uncertainty. We are not yet able to include all possible factors in the analysis, since there is no readily available dataset and method for parameterizing these complex processes. Additionally, we have visualized the impacts as a function of rain rate, however, the raindrop size and rainfall duration can impact the intensity of effects. It is known that larger raindrops can modify surface roughness more significantly, see e.g. [34]. The raindrop size also controls the terminal velocity and, consequently, the downdraft intensity, see [10] and references therein. At low wind speeds such as those in the analysis here, small wave damping in the low-frequency band of wave spectrum might happen whose rate could increase as rain duration prolongs [35].

Besides, technical limitations should be considered. Here, the measurement noise might degrade the retrieval accuracy. A noise power analysis is carried out in [23] using the same dataset. Noise power is calculated as the variance of quadrature component of the zenith looking antenna over a temporal segment. Accordingly, the average noise power is in general between -1 to $0 \mathrm{~dB}$, depending on the elevation angle. However, the standard deviation of the noise, varying between 2.5 and $3.5 \mathrm{~dB}$, depending again on the elevation angle, is large enough to affect the retrieval.

Finally, we rely here on physical models by calculating the difference between observed and modeled powers in Equation 11. Any inaccuracies in the models could also affect the retrieval efficiency. According to Equation 10, the surface roughness is supposed to have a polarization-independent behavior. The empirical data demonstrate inconsistencies since the RHCP observations show a lower level of sensitivity to the roughness change compared to LHCP observations. The existence of this dependency is shown in Figures 11 and 13. Figure 15 shows the discrepancies between the modeled and observed LHCP to RHCP power ratio. According to the theoretical model, the ratio is supposed to be roughness-free, but here the roughness effect is evident, which is larger in magnitude at high elevation angles. The model has not been able to sufficiently describe the polarization dependent results found e.g. by [23] and [33] neither. Besides, the challenges on modeling and correcting the roughness effects to retrieve soil moisture is discussed by [36]. There is still need and room for investigations on this topic.

This study has been focused on rain caused modifications of reflecting surface properties. Intense rainfalls can additionally induce depolarization effects on the signals propagating through the atmosphere in grazing angle geometry due to the asymmetry between the horizontal and vertical dimensions of the big droplets. This effect has been studied in Radio Occultation [13], [37] and ground-based direct measurements [38]. The change in the ellipticity of the incoming circularly polarized signals could in turn affect the power estimations at intense rains and low elevation angles. In contrast, the studies here show that the observed signatures are more intense at high elevation angles and also noticeable at low rain rates. Although this implies that the ellipticity change of the incoming signal could not be the dominant type of effect causing the signatures observed here, we encourage characterizing this type of effect in future studies. There are still important questions on how thereby the GNSS-R observations are affected requiring decent investigations using optimized setups and analysis constraints for this purpose. Such an investigation is beyond scope of this paper. The potential consequences in polarimetric GNSS-R measurements could be carried out in future studies with long enough datasets especially with dense measurements at high rain rates.

\section{SUMMARY AND DISCUSSION}

We investigated the response of polarimetric GNSS-R observations to precipitation for the first time. A discussion was made on the theoretical potential and the necessity of dual-polarization measurements for tracking the rain-caused SSS change in addition to sea surface modifications. The combination of the RHCP and LHCP observations amplifies the left signatures in the power ratios by the SSS change. Using a rain impact model, the change in the signal power due to 

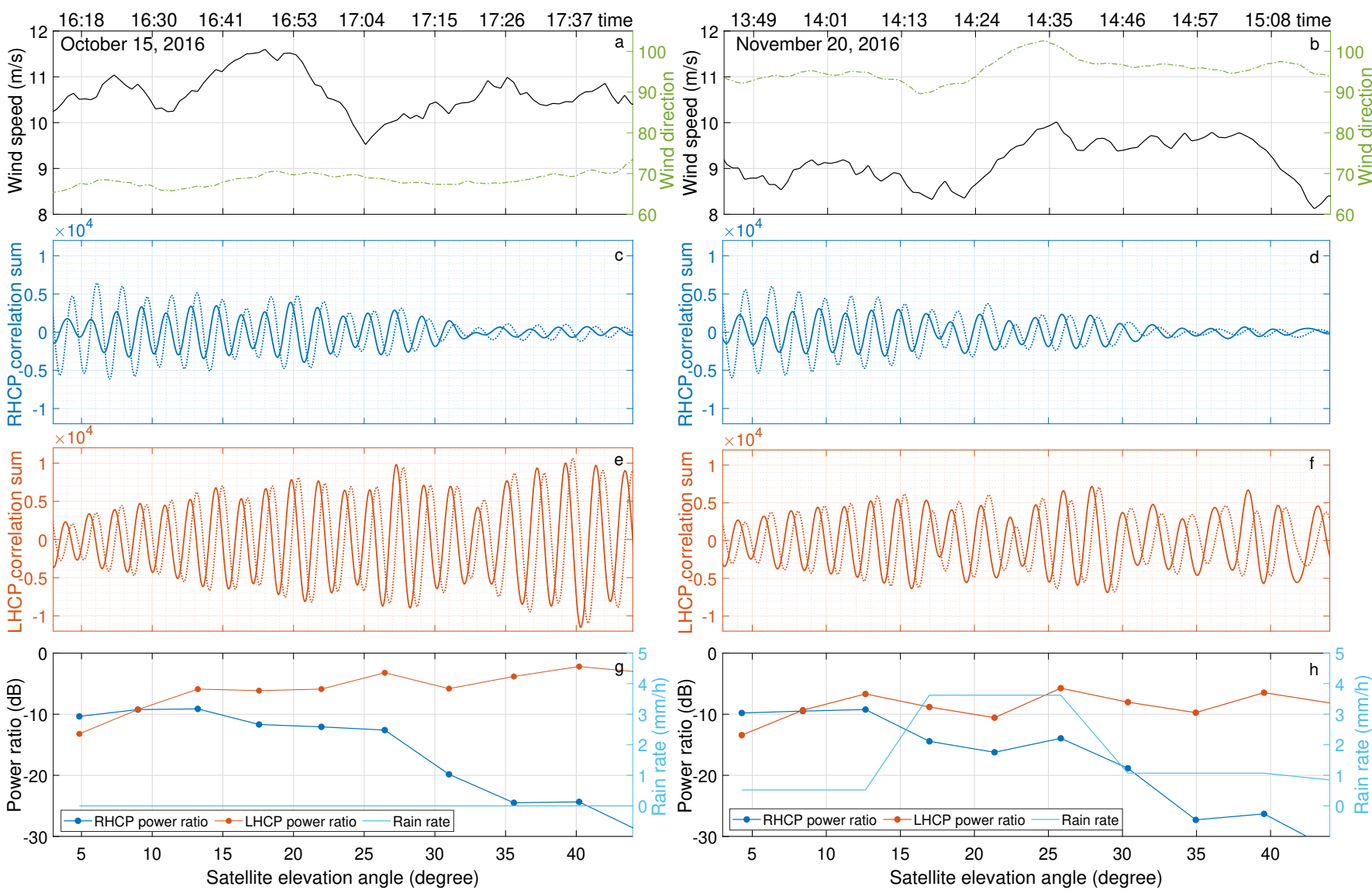

Fig. 13: An exemplary case of the rain impact on reflectometry observations from GPS satellite PRN 7. The graphs on the left side are related to the observations over a rain-free period. Right panels are associated with a time span that includes precipitation records based on the Global Precipitation Measurements (GPM) data. The reflectometry observations on both sides are selected to be over the same elevation angles of the satellite: Wind speed and direction $(\mathrm{a}, \mathrm{b})$, the receiver output (correlation sums), quadrature in dotted and in-phase in solid line, from the Right-Handed Circular Polarization (RHCP) antenna (c,d), the receiver output (correlation sums), quadrature in dotted and in-phase in solid line, from the Left-Handed Circular Polarization (LHCP) antenna (e,f), the ratios of the reflection power to direct signal power estimated from the RHCP and LHCP correlation sums against rain rate $(\mathrm{g}, \mathrm{h})$.

SSS change is simulated which shows a considerable impact at high rain rates with long enough duration.

An analysis is carried out using measurements of a coastal GNSS-R station with two (RHCP and LHCP) side-looking antennas. The rain effects result in the discrepancy of the average power ratios in cases during rainfall and at no rain, which is proportional to the elevation angles. The geometrydependency of the discrepancy magnitude implies a higher probability that the observed effects are mainly associated with the roughness change.

The left signature in both LHCP and RHCP power is large enough to be distinguishable in the measurements. The average LHCP power at an elevation angle of $45^{\circ}$ drops by $\approx 5 \mathrm{~dB}$, however, a significant increase in the standard deviation of the LHCP power is observed. In general, the LHCP observations enjoy a higher level of sensitivity to rain events, compared to RHCP measurements. This fact is further approved by investigating the amplitude of correlation sums in exemplary cases. The amplitude of the LHCP correlation sum shows more evident dampening by rainfall. This is a piece of evidence that the roughness loss is polarization-dependent, contrary to what the theoretical model implies. As a result, the models, especially that describing the power loss by the surface roughness, could be subjected to refinement in future studies.

An algorithm retrieving the SSS and surface state using the polarimetric measurements is proposed. The retrieved standard deviation of surface heights and the SSS also confirms that the general significant modification of the surface state, whereas, the SSS shows no meaningful behavior responding to rainfalls with rates below $10 \mathrm{~mm} / \mathrm{h}$. The retrieved SSS demonstrates a drop at higher rain rates. However, the data in this range is too sparse and studies with substantially higher number of observations at extreme rain rates are encouraged and still required.

The reported roughness change by rain splash in this study is substantially larger than the modifications described by models derived based on laboratory measurements. We discussed that this can be due to the inefficiency of these models in describing the altered roughness in a real sea. The results show an agreement with recent in situ measurements in the 

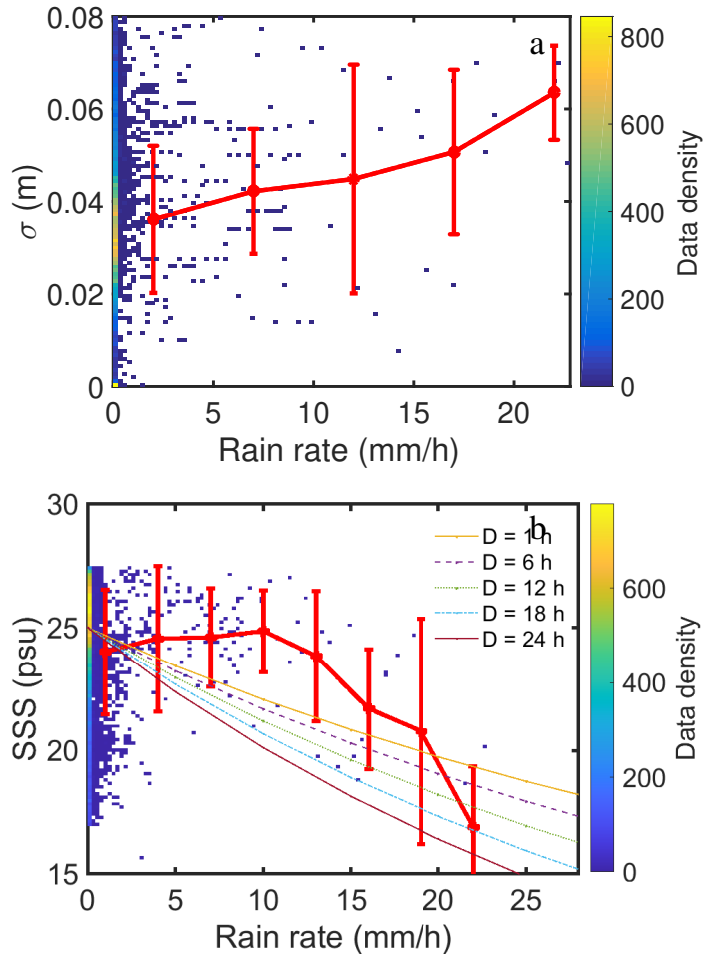

Fig. 14: Obtained values of standard deviation of sea surface heights $\sigma$ (a) and Sea Surface Salinity (SSS) along with simulated SSS at different rain duration $D$ (b) versus rain rate. The average values and standard deviations are shown in red.

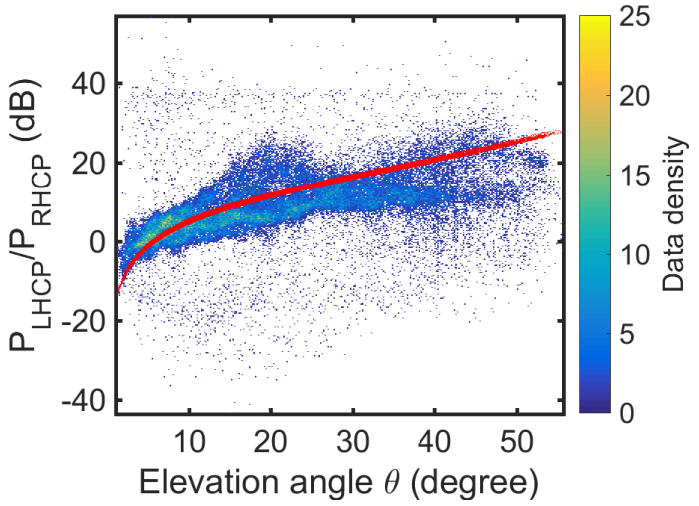

Fig. 15: The LHCP to RHCP power ratio vs. elevation angle along with simulations shown in red. The SST values are obtained from a nearby meteorological station. The SSS for each observation is estimated as an average value based on historical records of between 2001 and 2009 at a station located about 29 kilometers away. real environment. This also explains the quantitative mismatch between the effects seen in the spaceborne measurements and model-based simulations in the previous studies.

None of the datasets used so far for the investigation of rain sensitivity are optimized for this purpose. Future investigations could be carried out on datasets from GNSS$\mathrm{R}$ altimetry instruments due to the similarities of required environmental conditions, i.e. coherent reflections from calm surfaces. However, there is a dissimilarity on the desired reflection geometry. We are here interested in reflections at high elevation angles rather than those at grazing angles, considering the significant role of roughness change. The reflecting sea in the area of this study has smaller SSS values compared to global oceans remaining below 30 psu most of the time. In future studies on environments with higher SSS, SST change due to cold rainfalls can also be a significant research question over oceans with SSTs above $15^{\circ} \mathrm{C}$. The change in the ellipticity of the incoming circularly polarized signals propagating the rainy atmosphere deserves attention in future studies. With the derived knowledge in this study and previous investigations, we encourage future experiments fully dedicated to this topic.

\section{ACKNOWLEDGEMENTS}

We would like to thank Dr. Georg Beyerle at the German Research Centre for Geoscineces GFZ for his contributions in this study and the Onsala Space Observatory (OSO) for hosting the GNSS Reflectometry station. We also acknowledge the Swedish Meteorological and Hydrological Institute (SMHI) and the team associated with the NASA's Global Precipitation Measurement Mission (GPM) for the ancillary data used in this investigation.

\section{REFERENCES}

[1] Simon Michael Papalexiou and Alberto Montanari. Global and regional increase of precipitation extremes under global warming. Water Resources Research, 55(6):4901-4914, 2019.

[2] George J Huffman, David T Bolvin, Eric J Nelkin, David B Wolff, Robert F Adler, Guojun Gu, Yang Hong, Kenneth P Bowman, and Erich F Stocker. The TRMM multisatellite precipitation analysis (TMPA): Quasiglobal, multiyear, combined-sensor precipitation estimates at fine scales. Journal of hydrometeorology, 8(1):38-55, 2007.

[3] Arthur Y Hou, Ramesh K Kakar, Steven Neeck, Ardeshir A Azarbarzin, Christian D Kummerow, Masahiro Kojima, Riko Oki, Kenji Nakamura, and Toshio Iguchi. The global precipitation measurement mission. Bulletin of the American Meteorological Society, 95(5):701-722, 2014.

[4] Qiaohong Sun, Chiyuan Miao, Qingyun Duan, Hamed Ashouri, Soroosh Sorooshian, and Kuo-Lin Hsu. A review of global precipitation data sets: Data sources, estimation, and intercomparisons. Reviews of Geophysics, 56(1):79-107, 2018

[5] Valery U Zavorotny, Scott Gleason, Estel Cardellach, and Adriano Camps. Tutorial on remote sensing using GNSS bistatic radar of opportunity. IEEE Geoscience and Remote Sensing Magazine, 2(4):8-45, 2014.

[6] Christopher S Ruf, Clara Chew, Timothy Lang, Mary G Morris, Kyle Nave, Aaron Ridley, and Rajeswari Balasubramaniam. A new paradigm in earth environmental monitoring with the CYGNSS small satellite constellation. Scientific reports, 8(1):1-13, 2018.

[7] Milad Asgarimehr, Valery Zavorotny, Jens Wickert, and Sebastian Reich Can GNSS reflectometry detect precipitation over oceans? Geophysical Research Letters, 45(22):12-585, 2018.

[8] Alexander G Voronovich and Valery U Zavorotny. The transition from weak to strong diffuse radar bistatic scattering from rough ocean surface. IEEE Transactions on Antennas and Propagation, 65(11):6029-6034, 2017. 
[9] Adriano Camps, Jordi Font, Mercè Vall-llossera, I Corbella, N Duffo, F Torres, S Blanch, A Aguasca, R Villarino, Carolina Gabarró, et al. Determination of the sea surface emissivity at L-band and application to SMOS salinity retrieval algorithms: Review of the contributions of the UPC-ICM. Radio Science, 43(03):1-16, 2008.

[10] Rajeswari Balasubramaniam and Christopher Ruf. Characterization of rain impact on L-Band GNSS-R ocean surface measurements. Remote Sensing of Environment, 239:111607, 2020.

[11] Estel Cardellach, Yang Nan, Weiqiang Li, Ramon Padullés, Serni Ribó, and Antonio Rius. Variational Retrievals of High Winds Using Uncalibrated CyGNSS Observables. Remote Sensing, 12(23):3930, 2020

[12] Milad Asgarimehr, Jens Wickert, and Sebastian Reich. Evaluating impact of rain attenuation on space-borne GNSS reflectometry wind speeds. Remote Sensing, 11(9):1048, 2019.

[13] Estel Cardellach, Sergio Tomás, Santi Oliveras, Ramon Padullés, Antonio Rius, Manuel de la Torre-Juárez, Francis Joseph Turk, Chi O Ao, E Robert Kursinski, Bill Schreiner, et al. Sensitivity of PAZ LEO polarimetric GNSS radio-occultation experiment to precipitation events IEEE Transactions on Geoscience and Remote Sensing, 53(1):190-206, 2014.

[14] Felipe G Nievinski and Kristine M Larson. Forward modeling of GPS multipath for near-surface reflectometry and positioning applications GPS solutions, 18(2):309-322, 2014.

[15] Valery U Zavorotny and Alexander G Voronovich. Scattering of GPS signals from the ocean with wind remote sensing application. IEEE Transactions on Geoscience and Remote Sensing, 38(2):951-964, 2000.

[16] Lawrence Klein and C Swift. An improved model for the dielectric constant of sea water at microwave frequencies. IEEE transactions on antennas and propagation, 25(1):104-111, 1977.

[17] Thomas Meissner and Frank J Wentz. The complex dielectric constant of pure and sea water from microwave satellite observations. IEEE Transactions on Geoscience and remote Sensing, 42(9):1836-1849, 2004.

[18] Yiwen Zhou, Roger H Lang, Emmanuel P Dinnat, and David M Le Vine. L-band model function of the dielectric constant of seawater. IEEE Transactions on Geoscience and Remote Sensing, 55(12):6964-6974, 2017.

[19] C Ruf, P Chang, MP Clarizia, S Gleason, Z Jelenak, J Murray, M Morris, S Musko, D Posselt, D Provost, et al. CYGNSS handbook. Ann Arbor MI, Michigan Pub, 154, 2016.

[20] David M Le Vine and Emmanuel P Dinnat. The Multifrequency Future for Remote Sensing of Sea Surface Salinity from Space. Remote Sensing, 12(9):1381, 2020

[21] Andrea Santos-Garcia, María Marta Jacob, W Linwood Jones, William E Asher, Yazan Hejazin, Hamideh Ebrahimi, and Monica Rabolli. Investigation of rain effects on Aquarius sea surface salinity measurements. Journal of Geophysical Research: Oceans, 119(11):7605-7624, 2014.

[22] Magdalena D Anguelova and Peter W Gaiser. Skin depth at microwave frequencies of sea foam layers with vertical profile of void fraction Journal of Geophysical Research: Oceans, 116(C11), 2011.

[23] M. Hoseini, M. Semmling, H. Nahavandchi, E. Rennspiess, M. Ramatschi, R. Haas, J. Strandberg, and J. Wickert. On the response of polarimetric gnss-reflectometry to sea surface roughness. IEEE Transactions on Geoscience and Remote Sensing, pages 1-12, 2020. (Early Access).

[24] Arthur Mason Worthington. A study of splashes. Longmans, Green, and Company, 1908

[25] LF Bliven, PW Sobieski, and Christophe Craeye. Rain generated ringwaves: measurements and modelling for remote sensing. International Journal of Remote Sensing, 18(1):221-228, 1997.

[26] LF Bliven, Hubert Branger, Piotr Sobieski, and Jean-Paul Giovanangeli. An analysis of scatterometer returns from a water surface agitated by artificial rain: evidence that ring-waves are the main feature. International journal of remote sensing, 14(12):2315-2329, 1993.

[27] Ying-Keung Poon, Shih Tang, and Jin Wu. Interactions between rain and wind waves. Journal of Physical Oceanography, 22(9):976-987, 1992.

[28] Christophe Craeye, PW Sobieski, and LF Bliven. Scattering by artificial wind and rain roughened water surfaces at oblique incidences International Journal of Remote Sensing, 18(10):2241-2246, 1997.

[29] Nathan JM Laxague and Christopher J Zappa. The impact of rain on ocean surface waves and currents. Geophysical Research Letters, 47(7):e2020GL087287, 2020.

[30] Achim Helm, Georg Beyerle, Ralf Stosius, Oliver Montenbruck, Sergej Yudanov, Markus Rothacher, et al. The GNSS occultation, reflectometry, and scatterometry space receiver GORS: Current status and future plans within GITEWS. In Proceedings of the ESA 1st Colloquium, Scientific and Fundamental Aspects of the Galileo Programme, Toulouse, France, pages $1-4,2007$.
[31] AM Semmling, Jens Wickert, S Schön, Ralf Stosius, Markus Markgraf, Thomas Gerber, Maorong Ge, and Georg Beyerle. A zeppelin experiment to study airborne altimetry using specular Global Navigation Satellite System reflections. Radio Science, 48(4):427-440, 2013.

[32] Kristine M Larson, Eric E Small, Ethan Gutmann, Andria Bilich, Penina Axelrad, and John Braun. Using GPS multipath to measure soil moisture fluctuations: initial results. GPS solutions, 12(3):173-177, 2008.

[33] A Maximilian Semmling, Anja Rösel, Dmitry V Divine, Sebastian Gerland, Georges Stienne, Serge Reboul, Marcel Ludwig, Jens Wickert, and Harald Schuh. Sea-Ice Concentration Derived From GNSS Reflection Measurements in Fram Strait. IEEE Transactions on Geoscience and Remote Sensing, 57(12):10350-10361, 2019.

[34] A note on surface waves due to rain, author=Houk, D and Green, T. Journal of Geophysical Research, 81(24):4482-4484, 1976.

[35] R Rajesh Kumar, B Prasad Kumar, and D Bala Subrahamanyam. Parameterization of rain induced surface roughness and its validation study using a third generation wave model. Ocean Science Journal, 44(3): 125-143, 2009.

[36] Adriano Camps, Hyuk Park, Jordi Castellví, Jordi Corbera, and Emili Ascaso. Single-pass soil moisture retrievals using gnss-r: Lessons learned. Remote Sensing, 12(12):2064, 2020.

[37] E Cardellach, S Oliveras, A Rius, S Tomás, CO Ao, GW Franklin, BA Iijima, D Kuang, TK Meehan, R Padullés, et al. Sensing heavy precipitation with GNSS polarimetric radio occultations. Geophysical Research Letters, 46(2):1024-1031, 2019.

[38] Hao An, Wei Yan, Shuangshuang Bian, and Shuo Ma. Rain Monitoring with Polarimetric GNSS Signals: Ground-Based Experimental Research. Remote Sensing, 11(19):2293, 2019.

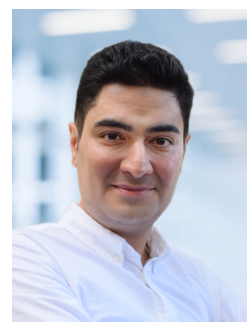

Milad Asgarimehr received his M.Sc. degree in Geodesy from K. N. Toosi University of Technology, Tehran, Iran, in 2015. Being awarded a PhD Fellowship by Geo.X, the geoscientific competence network in Berlin and Potsdam, he joined the German Research Centre for Geosciences GFZ, Potsdam, and Technische Universität Berlin, Germany, in 2017 His $\mathrm{PhD}$ dissertation was dedicated to the GNSS Reflectometry and remote sensing of the ocean and atmosphere. He was awarded the Bernd Rendel Prize in Geosciences by the German Research Foundation DFG and defended his PhD in 2020 with the highest distinction. He is currently a postdoctoral researcher at GFZ with research interests mainly in bistatic radar, development of novel remote sensing techniques, and studying Earth systems using the derived data especially with the help of artificial intelligence.

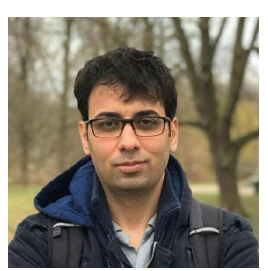

Mostafa Hoseini graduated in Geodesy from the University of Tehran, Iran. He worked for several institutions as a GNSS engineer in the field of positioning and navigation. Before starting his $\mathrm{PhD}$ studies in 2018 at the Norwegian University of Science and Technology (NTNU), he worked on the analysis of GNSS atmospheric products at the German Research Centre for Geosciences GFZ in Potsdam, Germany. His research interests include GNSS-based sensors and techniques for remote sensing applications. His current research focuses on the monitoring of the ocean and Arctic using GNSS-Reflectometry onboard CubeSats. 


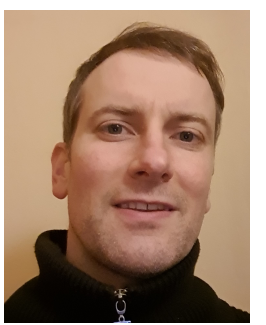

Maximilian Semmling graduated in physics from Leipzig University in 2007 and received the doctoral degree from Technische Universität Berlin in 2012. For his doctoral and post-doctoral studies, he was with the German Research Centre for Geosciences (GFZ), Germany, the Institute for Space Studies of Catalonia (IEEC), Spain, and the University of the Littoral Opal Coast (ULCO), France. In 2020 he joined the observation department at the German Aerospace Center (DLR), Institute for SolarTerrestrial Physics. He is experienced in GNSS for Earth Observation with research focus on ocean altimetry and sea ice remote sensing. His current work concentrates on the influence of atmosphere and space weather. Corresponding methods cover a wide range from ground-based measurements over maritime, airborne to satellite platforms.

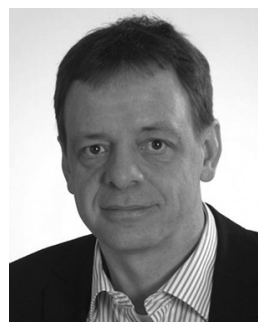

Markus Ramatschi graduated in geophysics in 1992 and received a Ph.D. degree in geophysics from the Technical University of Clausthal, ClausthalZellerfeld, Germany, in 1998. Since 2000 he is a senior scientist at GFZ Potsdam, Potsdam, Germany. His work focuses mainly on the operation of GNSS sensor stations and related hardware developments.

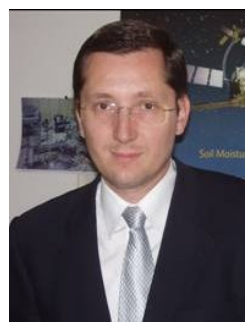

Adriano Camps (Fellow IEEE 2011) was born in Barcelona, Spain, in 1969. In 1993 he joined the Electromagnetics and Photonics Engineering Group, Department of Signal Theory and Communications, UPC, as an Assistant Professor, Associate Professor in 1997, and Full Professor since 2007. In 1999, he was on sabbatical leave at the Microwave Remote Sensing Laboratory, of the University of Massachusetts, Amherst. His research interests are focused in microwave remote sensing, with special emphasis in microwave radiometry by aperture synthesis techniques (MIRAS instrument onboard ESA's SMOS mission), remote sensing using signals of opportunity (GNSS-R), and nanosatellites as a tool to test innovative remote sensors. He has published over 223 papers in peer-reviewed journals, 8 book chapters and the book Emery and Camps, "Introduction to Satellite Remote Sensing. Atmosphere, Ocean, Land and Cryosphere Applications," Elsevier, 2017, 860 pages), and more than 471 conference presentations, holds 12 patents, and has advised $27 \mathrm{Ph}$. D. Thesis students (+ 8 on-going), and more than 140 final project and M.Eng. Theses. According to Google Scholar/Scopus his h-index is 52/40. He has received several awards, among which the European Young Investigator Award (2004), the ICREA Academia research award $(2009,2015)$, and the Duran Farell award for Technology Transfer $(2000,2010)$.

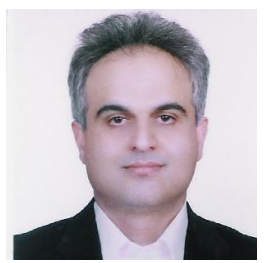

Hossein Nahavandchi received the Ph.D. degree from the Royal Institute of Technology (KTH), Stockholm, Sweden in 1998. He is currently a Professor of Geodesy and Geophysics with the Norwegian University of Science and Technology (NTNU), Trondheim, Norway. His research interests involve Global Geodetic Observations System (GGOS) including satellite gravimetry, satellite altimetry, satellite radar interferometry and GNSS to understand the dynamic Earth system by quantifying Earth's change in space and time.

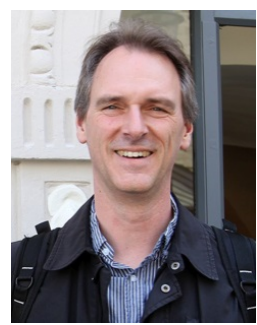

Rüdiger Haas is a full professor for space geodesy at the Department of Space, Earth and Environment at Chalmers University of Technology, Sweden. He received his $\mathrm{MSc}$ and his $\mathrm{PhD}$ degrees in geodesy from Bonn University, Germany. He is the head of the research group for space geodesy at Chalmers and responsible for the geoscience activities at the Onsala Space Observatory. His research interests focus primarily on space geodetic techniques, such as Global Navigation Satellite Systems (GNSS), inInterferometry (VLBI).

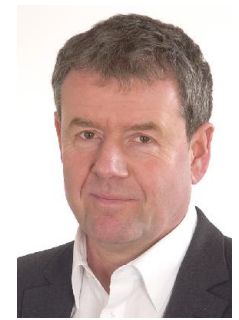

Jens Wickert received the Diploma degree in physics from Technical University Dresden, Dresden, Germany, in 1989, and the Ph.D. degree in geophysics/meteorology from Karl-Franzens University Graz, Graz, Austria, in 2002. He was the Principal Investigator of the pioneering GPS radio occultation experiment aboard the German CHAllenging Minisatellite Payload (CHAMP) Satellite and coordinates numerous research projects on GNSS remote sensing. He holds a joint professorship of GFZ with the Technical University of Berlin on Global Navigation Satellite Systems (GNSS) remote sensing, navigation, and positioning. He is also the Deputy GFZ Section Head of Space Geodetic Techniques and the GFZ Research Topic Director of The Atmosphere in Global Change. He has authored or coauthored more than $250 \mathrm{Web}$ of Science indexed publications on GNSS Earth observation. 